\title{
Homogeneous and Heterogenious Traffic Conditions Modellingof A Route Choice Network System
}

\author{
Sabbidi Venkatesham, Santhosh Kumar
}

\begin{abstract}
Demonstrating course decision conduct is dangerous, yet basic to evaluate explorers' impression of course attributes, to estimate voyagers' conduct under theoretical situations, to anticipate Future site visitors situations on transportation systems and to comprehend voyagers' response and adjustment to wellsprings of information. We increase a perceptible heterogeneous traffic flow version. This version considers trade of various vehicle lessons, each one of that's anticipated to have homogeneous vehicle following conduct and automobile trends. We advocate the thoughts of road restrict break up and saw same thickness for every elegance to expose both horizontal and longitudinal go-class collaborations crosswise over neighboring cells. Instead of making use of hydrodynamic analogies, it builds up sensible go-class verbal exchange concepts sought through restrict distribution and surmised between mobile motions.
\end{abstract}

The congestion phenomenon is considered in desk bound situations via the conduct ofamounts: the suggest tour time of a parcel and the mean quantity of bundles that have no longer arrived at their cause and are travel-ing in the system. We characterize a exchange that maps a gadget having the little global belongings (Inet 3037 in our numerical examinations) into an (altered) grid arrange that has a comparable range of hubs. This guide adjustments the restrict of the additives of the charts speakme to the systems and can be considered as an "interjection" among the two instructions of systems. Utilizing this change we evaluation the conduct of Inet 3037 with the behavior of an altered rectangular grid and we have a look at the conduct of the placing systems. This examination recommends the way to alternate the system topology and the department limits so that it will mitigate the congestion phenomenon.Understanding the causes and dynamics of nonequilibrium traffic flow via models, observations, and data is a crucial challenge in traffic flow theory; and it becomes even more important in light of ongoing and future (partial) automation of the vehicle fleet. We highlight important steps in developing phenomenological models for instabilities and nonlinear waves in traffic flow, both macroscopic and microscopic, and discuss how this modeling extends to heterogeneous flows composed of a mix of human-driven and (semi-)automated vehicles. In particular, we stress how automation can go both ways: it could make traffic run more smoothly; but also render it less efficient than it is under human control.

Keywords: Inet, Semi automated, Automated Etc.

\section{INTRODUCTION}

Traffic movement elements is managed by way of targeted visitors floods of numerous characters, which come from each heteroge-neous vehicle factors and driver

Revised Manuscript Received on August 19, 2019.

SabbidiVenkatesham, Research scholar department of civil engineering University of technology, jaipur. Jaipur

Santhosh Kumar, Professor In Civil Dept, University Of Technology, tendencies. For instance, in a multi-modular framework, traveller motors, travel armadas and trucks proportion the option to proceed. The distinctive vehicle characters essentially bless them with heterogeneous safety dis-tances, wanted paces, and paths to go on. As every other version, drivers (counting each human and mechanized computerized drivers) can show heterogeneous driving conduct, e.G. holes to keep when following the main automobiles, recurrence to apply brakes, and propensity of route evolving, and so on. This further entangles the total traffic elements. While het-erogeneity is a universal character of traffic stream in reality, various investigations demonstrate that reproducible thickness stream relations (for example central graphs) Can be recreated while is a visitors circulation is homogeneous and desk bound ( Cassidy, 1998; Laval and Chilukuri, 2016; Jin, 2017 ). Along these strains, it's far realistic to expect that a homogeneous site visitors circulate can at present be all round depicted by way of a properly-characterised (nonstop and inward) most important chart. To further show what the above presumption indicates, we remember a visitors stream comprising of motors following indistinguishable probabilistic automobile following hints, which are portrayed by the outstanding Nagel-Schreckenberg mobile automaton (CA) version ( Nagel and Schreckenberg, 1992; Tian et al., 2016 ). The happened circulation thickness connection is all around approximated by means of a triangle connection, as we seem in Fig. 1 . This parent delineates the scenario where we reenact site visitors movement in a solitary route circle comprising of in every of the 100 cells, with the quantity of cars transferring from 50 to 550 motors.

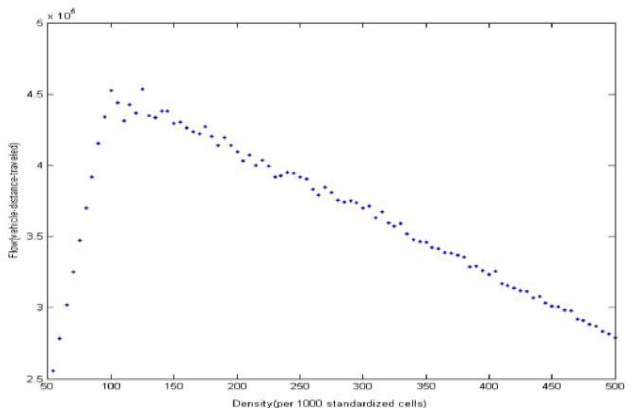

Fig. 1.A fundamental diagram from NagelSchreckenberg traffic. 
Course decision models help dissecting and understanding explorers' conduct, yet in addition establish the fundamental piece of traffic task strategies. In the deterministic person equilibrium(DUE) trouble, a straightforward path selection version accept ridiculously that explorers have perfect mastering about way fees and pick out the route that limits their movement expenses. In the stochastic patron equilibrium (SUE) problem, a probabilistic direction desire version take delivery of sensibly that explorers have incorrect information about manner expenses and pick out the direction that limits their obvious tour fees given a lot of publications. In particular dynamic site visitors task(DTA), a route selection model is both foreordained or registered even as the system stacking instrument works.

In this paper we have a look at the facts package site visitors clog wonder in transmission organizes resulting from Ho-mogeneous and heterogeneous site visitors. This is an enthusiasm ing surprise with a few right down to earth applications amongst which we word the studies of the behavior of media transmission systems and specially of the be-haviour of Internet. More when all is stated in performed the clog expert blem in machine streams is of tremendous enthusiasm for some en-gineering fields, consisting of, pipeline move, electric energy transmission [1], broadcast communications and high-approaches or railroads traffic the executives. To repair the thoughts allow us to take into account the Internet traffic. The Internet internet-work develops in length every day and its attainable clog is an relevant difficulty from the social and the financial point

Of view. Truth be advised it's far a few factor however tough to image the damage that the Internet blockage will purpose to the social members of the family and to the financial exchanges that stand up on it. In the maximum recent years some stop-has had been delivered to the brink of the Internet set up and those new passages have inwrinkled generously the heap of thesystem.

\section{LITERATURE REVIEW}

De la Barra et al. (1993) present the connection punishment heuristic, which develops the decision set by iteratively putting away ways. The main course put away is the real most brief way, and the extra put away ways are the most brief ways processed after impedances on connections having a place with the past way are expanded by a fixed rate. The method closes when two put away courses agree. Ruphail et al. (1995) symbolize the Iterative Penalty Method (IPM), in which the punishment might be linked no longer exclusively to the modern-day briefest manner, but to all the maximum restrained methods decided, by means of both inclusive of a hard and fast impedance sum or duplicating their impedances through a fixed time period. Park and Rilett (1997) recommend a variant of the first manner to deal with abstain from handing over minor deviations toward the start or the part of the good deal. In particular, impedances on connections aren't improved internal a selected accurate approaches from the beginning or the aim of the excursion. Subsequently, created approaches are less comparative and the significance of the courses increments. Scott et al. (1997) develop the technique with a streamlining software for determining the punishment component for briefest manner connects as a way to create a subsequent maximum restrained way that covers with the present one by means of close to a given range of connections.

Bekhor et al. (2006) symbolize punishment elements similar to the separation amongst root and aim of the watched guides, so better augmentations take place for longer removes. Prato and Bekhor (2006) distinguish an interesting punishment factor and emphasize the method multiple times. The two investigations demonstrate that connection punishment does not give improvement regarding age of significant courses, as the strategy duplicates somewhat second rate rates of watched courses as for the connection end approach.

The upsides of connection punishment are the prospect for fundamental connects to stay in the system and the demoralization for effectively recognized connects to have a place with most limited ways In similarly cycles. The regulations of this system comprise within the age of high impedance methods that undermines the importance of the guides and inside the reliance on the which means of the punishment element. With low traits the calculation isn't computationally efficient as a similar manner is recognized over and over, while with excessive features high impedance and unpleasant methods are created earlier than shorter and gradually appealing ones.

Nielsen (2000) separates arbitrary draws from a truncated normal in an research concerning the Copenhagen-Ringsted railroad venture. Smashing (2002) eliminates forty eight draws from a ordinary conveyance, with mean and distinction equal to the connection adventure time. Bierlaire and Frejinger (2005) separate 20 draws from a truncated commonplace appropriation, with suggest and distinction equivalent to the relationship excursion times recorded by using way of GPS devices at some stage in a site visitors security study inside the Swedish metropolis of Borlange. Prato and Bekhor (2006) separate 35 attracts from a truncated common dissemination, with suggest and fluctuation same to the relationship adventure time, left truncation restriction equal to the unfastened-flow time and proper truncation restriction equivalent to the motion time determined for a base pace predicted equivalent to ten $\mathrm{km} / \mathrm{h}$.

Smashing (2002) separates various draws that contains the computational expenses inside similar limits of connection end and connection punishment draws near, As the computational intake of the pastime approach is surely relative to the amount of draws. Note that the augmentation in the quantity of attracts altogether expands the computational charges, however does no longer make sure the age of extra novel approaches. Prato and Bekhor (2006) separate various draws that depletes the capacity of the technique to create interesting ways, as the standard deviation confines the likelihood of delivering exceptionally various courses. With low estimations of the fluctuation the calculation isn't effective in light of the fact that not many one of a kind ways are created over and again, while with high qualities the methodology is additionally not proficient on the grounds that various ridiculous ways are delivered. 


\section{ROUTE CHOICE MODELS}

\subsection{The problem}

Subsets of non-obligatory courses produced with the portrayed manner age methods are generally very vast for each model estimation and circulate forecast, since all the important courses are conceivably protected and some immaterial courses are most in all likelihood made. Contextual analyses in European and American city systems show that as much as 70 alternatives are considered as across the board approach to assess discrete selection models, and up to a hundred choices are considered as ordinary exercise To carry out site visitors challenge. Instinctively, the quantity of options within the selection set assumes a task inside the estimation of discrete preference fashions in the direction choice setting.

Late investigations supply information into the selection set effect on version opinions and exhibitions. Bekhor et al. (2006) examine the effect of check duration in way based totally venture by using the usage of looking at the first-rate Winnipeg machine and demonstrating that larger preference sets improve combination and aim ability esteems. Prato and Bekhor (2007) display a crucial impact of length and sythesis of created route units on parameter gauges and version exhibitions, regardless of the truth that announced effects take a look at usual execution insights in vicinity of estimation incredible on the diploma of person desire units. Frejinger (2007) demonstrates that the whole decision set is critical to compute connection measures and get ideal estimation outcomes. Bliemer and Bovy (2008) deal with the problem of path selection forecast and display that the expansion of immaterial courses predispositions direction selection probabilities and makes appealing courses become much less eye-catching, conversely with the regular version heartiness in the direction of the growth of unimportant courses (Bovy, 2009).

In like manner, path selection models ought to display power in software parameter gauges regarding choice set size. For estimation functions, this version prerequisite could allow the meaning of selection units with a realistic wide variety of attractive alternatives for you to get stable model exams. For forecast purposes, this version prerequisite would no longer settle the issue of the need to create all the critical publications.

Thick urban structures with 70 or a hundred options demonstrate a high degree of likeness among elective courses. Hence, a large portion of the writing centers across the connection between's alternatives, which modifies choice chances of masking courses. Prashker and Bekhor (2004) delineate the basic cover problem, that is the course decision companion of the widely known pink delivery blue shipping trouble, and a basic direction converting issue to peer that few route choice fashions do not effectively capture the closeness among publications in each machine setup. Bliemer and Bovy (2008) keep in mind a Monte Carlo exercise of path choices from a matrix device to represent that guides with a similar duration aren't similarly favored, as the extra the publications cowl, the lower their likelihood for an increasing number of self sustaining alternatives. Plainly, path decision fashions must have the option to speak to accurately the relationship structure amongst optionally available courses. Regardless of whether the likeness is seen at the connection level or at the street various leveled level is not certain, and fashions equipped to contain each these views are exceptional. Note that the most famous discrete choice models within the act of movement behavior displaying, Multinomial Logit(MNL) and Nested Logit (NL), aren't appropriate to reveal path selection. MNL does now not allow to represent likeness amongst alternatives, while NL count on that each choice has an area..

\subsection{Logit structures}

MNL-alterations keep up the truthful Logit shape and present a treatment term in the deterministic piece of the software capability to hard the connection amongst optionally available guides.

\subsubsection{C-Logit}

Cascetta et al. (1996) suggest the number one trade of the MNL version, in which a shared feature element estimates the extent of comparison of each route with different courses in the decision set $\mathrm{C}$. The announcement of the probability $\mathrm{Pk}$ of choosing course ok in the choice set $\mathrm{C}$ mirrors the simple Logit shape of the version:

$$
P_{k}=\frac{\exp \left(V_{k}+\beta_{C F} \cdot C F_{k}\right)}{\sum_{l \in C} \exp \left(V_{l}+\beta_{C F} \cdot C F_{l}\right)}
$$

Points of interest of the C-Logit version include in an appropriate heartiness of utility parameter gauges as for the selection set length (Prato and Bekhor, 2007) and in the presence of a proportional clinical definition for the SUE difficulty (Zhou and Chen, 2003). The real disservices of the C-Logit version are that the shared characteristic element catches just piece of the closeness and choice standards for the definition of the shared function elements are not proposed. Contextual analyses give proof of irrational outcomes as far as the indication of the parameter $\beta \mathrm{CF}$ when the shared trait factor is processed by articulations (3) and (4), while represent expected and agreeable outcomes when the shared trait factor is registered by articulation (five) (Ramming, 2002; Prato, 2005; Prato and Bekhor, 2007). Further, opportunity esteems screen that the Path-Size Logit model thru and massive outflanks the C-Logit model (Ramming, 2002; Prato and Bekhor, 2006; Prato and Bekhor, 2007). In all probability this clarifies why present research about MNL-changes advances in the direction of new details of the way length revision time period in place of new articulations of the shared characteristic factor 


\section{TRAFFIC CONGESTION PHENOMENON IN THE HOMOGENEOUS AND HETEROGENEOUS TRAFFIC CASEES\& RESULTS}

\subsection{The Traffic Congestion Phenomenon in the Homogeneous Traffic Case}

Give us a chance to think about the blockage marvel in Thehomoge-neous site visitors case for the systems portrayed Via the weighted contiguousness lattices characterised in (11). Note that the homogeneous web site site visitors case may be received as an uncommon instance of the heterogeneous traffic case, as an instance, with the choice of the want factors: $\mathrm{p} 1=\mathrm{p} 2=\mathrm{p} 3=1$ or with the choice $\beta 1=1, \beta 2=\beta 3=$ zero. At that issue in the homogeneous traf-fic case the suggest heap of bundles age is identical to $\beta 1 \lambda=\lambda$. At time zero the structures pondered are vacant, this is they do no longer comprise facts bundles. This truth produces a brief conduct that interfaces the underlying nation (as an example void tool) to the massive time behavior of the web site traffic at the tool. For the homogeneous and the heteroge-neous web site traffic case the activity of the statistics bundle visitors is finished within the time meantime $[0, \mathrm{~T}]$. The time $\mathrm{T}$ is picked to be 4 hundred time units as a way to make sure that within the loose move device the visitors on the structures taken into consideration has (around) arrived on the desk bound situation. The reproduction system inside the homogeneous rush hour gridlock case accommodates of the accompanying advances:

1) Choose the quantity $\mathrm{N} \alpha+1$ of the machine topologies to be able to be taken into consideration inside the reproductions, we don't forget $\mathrm{N} \alpha=10$;

2) Choose the tool topologies, that is, relegate the estimations of $\alpha$ applied inside the reenactments, we bear in mind $\alpha=\alpha j=(j-1) / N \alpha, j=1,2, \cdots, N \alpha+1$;

3) Define the topology of the systems pondered, for $\mathrm{j}=$ $1,2, \cdots, \mathrm{N} \alpha+1$, we do not forget the gadget related to the weighted nearness grid $\mathrm{jA} \square$ characterized by the use of (eleven) when $\alpha=\alpha \mathrm{j}$, that is the $\alpha=\alpha \mathrm{j}$-put together topology;

4) Packets age: in whenever unit of the simula-tion we first example from a Poisson odd variable with suggest $\lambda$ the amount of parcels produced in that point unit. The estimations of $\lambda$ taken into consideration inside the recreations are 201 features equispaced inside the period in-between, this is we pick out $\lambda=\lambda \mathrm{k}$ hundred. A few hubs are doled out to each package produced. This couple of hubs is inspected from an arbitrary variable continually disseminated On the arrangement of couples (s, d), with the cease purpose that $\mathrm{s} \neq \mathrm{d}, \mathrm{s}, \mathrm{d}=1,2, \cdots, 3037$. For $\mathrm{s}, \mathrm{d}=1,2, \cdots$, $3037, s \neq d$, the bundle related to the couple $(s, d)$, begins offevolved inside the hub $\mathrm{s}$ and its purpose is the hub d;

5) Routing: the steerage comprises in partner to each parcel a briefest weighted way associating the hub in which the package starts offevolved with its goal hub. The bundle is sent along this manner during the time steps that pursue the season of its age. At the point when the most limited weighted way between the $=10+(3000-10)$ ok/two hundred, ok $=$ zero, $1, \cdots, 2$

source and goal hubs of a parcel isn't special the bundle pursues the briefest weighted way controlled by the calculation used to com-pute the steering of the bundles. At the point when the parcel arrives at its goal is expelled From the site visitors simu-lation;

6) Queue the executives: each hub has a line for each department leaving the hub, we take shipping of the strains to be conceivably unbounded. At the component while a package deal is produced in a hub or touches base at a hub, it's miles set up the precise line of the hub at some point of the time spent being conveyed in the direction of its goal alongside its course. The line the board standard is: first in first out. Whenever at least two bundles that need to continue with their course on a comparable branch arrive simulta-neously at a hub the one that is going first within the line is the parcel that has been created earlier than.

We observe that the parameter $\lambda$ is the imply estimation of the amount of bundles produced inside the complete arrange in a period unit, with the intention that given the manner that the foundation and the goal hubs ascribed to the parcels are examined from a consistently appropriated arbitrary variable and the way that there are $n$ hubs in the system we can presume that each hub typical creates $\lambda / n$ bundles in line with time unit.

Note that we have picked as directing procedure the briefest weighted way (see equation (3)) rather than the more regular most limited way. This decision is made so as to exploit the system multifaceted nature, that is of the little world property delighted in by Inet 3037 (and by the $\alpha$ systems, $\alpha \in(0,1))$, and of the way that the parts of Inet 3037 have limits running in a wide interim such that makes conceivable to exploit the little world property. Truth be told on account of Inet 3037 the utilization of the briefest way course will coordinate practically every one of the parcels through the hubs with higher between's centrality bringing about a lacking abuse of the branch ca-pacities and at last in rush hour gridlock clog. Note that in the altered rectangular cross section case (i.e.: $\alpha=1$ ), because of the decision of giving a similar ability to every one of the branches, the most brief way and the most brief weighted way directing methodologies match.

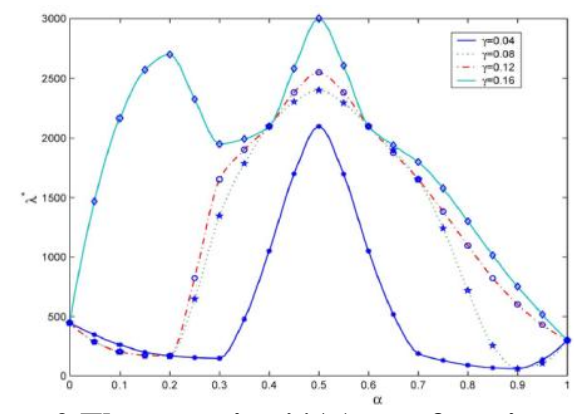

Figure 2.The quantity $\lambda *(\alpha)$ as a function of the parameter $\alpha$ for several choices of the threshold $\gamma$ (Criterion 1) 
In the homogeneous rush hour gridlock case allow NL, tM be respec-tively the portions corresponding to the quantitiesii,LM, I = 1, 2, three, offered already within the heterogenous rush hour gridlock case. Note that during NL, tM we drop the subscript I, due to the reality inside the homogeneous rush hour gridlock case there's only a single form of information bundles going on the gadget, this is we've got definitely $I=1$. The web page visitors reproduction demonstrates that, at the same time as there's no clog, after a brief time, whose term de-pends at the gadget topology, the website online traffic flow arrives at a desk bound kingdom in which, in regular, the all out type of parcels made and conveyed are equivalent, this is NL is a restricted amount and the propose journey time tM predicted in time gadgets is of the request for amount of the imply duration of the most brief weighted manner.

\subsection{The Traffic Congestion Phenomenon in the Heterogeneous Traffic Case}

Give us a hazard to ponder the blockage surprise in the heterogeneous site visitors case. As of now referenced inside the Introduction we study at the systems taken into consideration already the traffic of 3 numerous types of facts bundles. We do not forget three special site visitors instances appointing different esteems To the constants $\beta \mathrm{j}, \mathrm{j}$ $=1,2,3$, that characterizes the suggest burden $\beta \mathrm{i} \lambda$ of the parcels of kind $I, I=1,2$, three. That is we think about the accompanying instances:

Case (a): $\beta 1=\beta 2=\beta 3=1 /$ three. This is a decent load circumstance and produces a heap for every unit time having in regular a similar wide variety of parcels for the 3 sorts of bundles;

Case (b): $\beta 1=5 / 7, \beta 2=1 / 7, \beta 3=1 / 7$. This is an un-adjusted burden condition and produces a heap for every unit time having in normal an significant number of parcels with maximum improved want (type $I=1$ ), the stayingforms of bundles have in ordinary fewer bundles and in fashionable ticular for the reason that $\beta 2=\beta 3$ they've a comparable number of \%-kets;

Case (c): $\beta 1=1 / 6, \beta 2=2 / 6, \beta 3=$ three/6. This is the most extensively recognized burden circumstance, this is in which the num-ber of bundles consistent with unit time with the maximum noteworthy need (type $\mathrm{I}=1$ ) is (in normal) littler than the amount of parcels in line with unit time of middle want (kind $I=2$ ) and this last wide variety is (in ordinary) littler than the amount of bundles per unit time with least need (kind $\mathrm{I}=3$ ).

The reproduction approach inside the heterogeneous rush hour gridlock case is just like the reenactment device within the homogeneous rush hour gridlock case portrayed in Section 3 other than Step 4, bundle age, and Step 6, line the board, that must be adjusted to the new circumstance.

\section{CONCLUSIONS}

- Dynamic instabilities and traffic waves are fundamental features of (human) traffic flow.

- Humans do not intend to produce those waves. It is hard to change human driving.

- Even automated systems can have instabilities $(-\rightarrow$ ACC experiment).
- Vehicle automation and connectivity will change the flow dynamics. Need models to understand challenges of those heterogeneous human-AI cyber-physical systems, ideally before bad stuff happens.

- At the same time, well-controlled CAVs can, even in low numbers, remove traffic waves.

- This can have quite substantial wealth $(40 \%$ less fuel consumed) and health (70\% less NOx emissions) consequences

\section{REFERENCES}

1. Farina, A. Graziano, F. Mariani and F.Zirilli, "Pro- babilistic Analysis of Failuresin Power Transmission Networks and PhaseTransitions: A Study Case of a High VoltagePower Transmission Network," Journal ofOpti-mization Theory and Applications, Vol.139, No. 1, 2008, pp. 171199.doi:10.1007/s10957-008-9435-x

2. L. Kocarev and G. Vattay, "ComplexDynamics in Communication Networks,"Springer Verlag, Berlin, 2005.doi:10.1007/b94627

3. W. Aiello, F. Chung and L. Lu, "RandomEvolution in Massive Graphs," In: J. Abello,P. M. Pardalos and M. G. C. Resende, Eds.,Handbook of Massive Data Sets, KluwerAcademic Publisher, Norwell, 2002, pp. 97-122.

4. L. Barabasi and R. Albert, "Emergence ofScaling in Random Network," Science, Vol.286, No. 5439, 1999, pp. 509512.doi:10.1126/science.286.5439.509

5. S. Jamin and J. Winick, "Inet-3.0: InternetTopology Generator," University ofMichigan, Ann Arbor, 2002.

6. Y. Aumann and Y. Rabani, "An O(log k)Approximate MinCut Max-Flow Theoremand Approximation Algo-rithm," SIAMJournal on Computing, Vol. 27, No. 1,1998, pp. 291301.doi:10.1137/S0097539794285983

7. H. Okamura and P. Seymour,"Multicommodity Flows in Planar Graphs,"Journal of Combinatorial Theory B, Vol. 31,No. 1, 1981, pp. 75-81. doi:10.1016/S00958956(81)80012-3

\section{AUTHORS BIOGRAPHY}

SabbidiVenkatesham, Research scholar department of civil engineering University of technology, jaipur. He received B.tech 2014 from (Amina Institute of technology), JNTUH, Hyderabad, M.tech in 2016 from (pullareddy institute of technology) Hyderabad. Svnk00@ gmail.com ,

Dr. Santhosh Kumar, Professor In Civil Dept, University Of Technology, Jaipur. Scholastically advanced Educationist with rich scholarly and authoritative experience; offering a famous profession crossing more than 15 years in different Engineering Colleges. Achieve with sound reputation of inventive academic accomplishments. Master in arranging, sorting out, planning and checking a wide range of curricular and co-curricular exercises for the general advancement character of understudies and the board of the organization. Sabbidvenkat2017@gmail.com

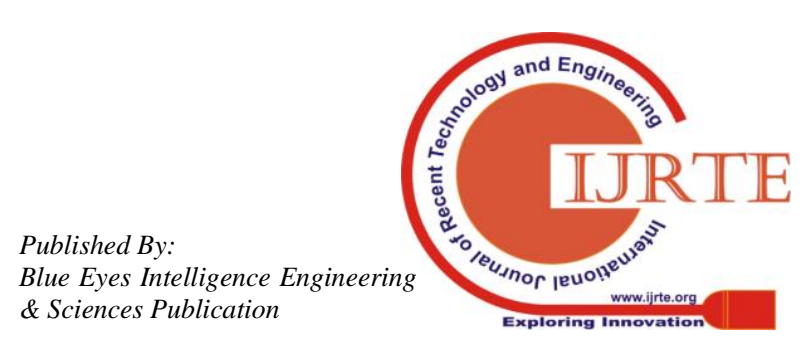

